\section{PSQ-163 MEDICATION RECONCILIATION IN AN EMERGENCY DEPARTMENT}

A Tomás Luiz*, M Almnchel Rivadeneyra, A Ruiz Gómez, M Saéz Garrido, 0 García Molina. Hospital Clínico Universitario Virgen de La Arrixaca, Pharmacy, Murcia, Spain

10.1136/ejhpharm-2019-eahpconf.596

Background Medication errors lead to higher morbidity, mortality and expenditure. The likelihood of mistakes is higher in the Emergency Department (ED).

Purpose To determine the incidence, the type of discrepancies and reconciliation errors (RE) upon admission to an ED, and the drugs involved.

Material and methods Prospective observational study, including patients admitted to the ED pending hospitalisation, during a period of 3 weeks (9-27 April 2018). The variables collected were: sex, age, number of home medications, number of discrepancies justified by the patient's clinical evolution (DJ) and not justified requiring clarification (DNJ), type of RE detected according to the Consensus Statement of the Spanish Society of Hospital Pharmacy and drugs involved. Programme coverage indicator, quality prescription indicators and medication reconciliation process indicators were calculated. The medication reconciliation process (MRP) was carried out through a clinical interview with the patient/carer, and the data obtained from the electronic clinical history and the primary care electronic records.

Results MRP was performed in 61 of the 216 patients admitted (coverage rate of 28.24\%). 55.74\% were males, with an average age of $70.61 \pm 14.86$ years $(72.13 \%>65$ years). The median of home medications was 8 (range 1-18). Ninety-three discrepancies were detected, of which $22.58 \%$ were DJ, while the remaining $77.42 \%$ were considered DNJ. The quality indicators of the prescription were determined, obtaining the following results: $57.38 \%$ patients with RE, $42 \%$ medications with RE and 1.20 RE per patient. Regarding quality indicators of the MRP, the detected RE were $58.33 \%$, and were classified into: 37 (88.10\%) medication omissions, four (9.52\%) dose errors, and one (2.38\%) wrong medication. The drugs involved were: 19 (45.24\%) lipid modifying agents, five (11.90\%) antidepressants, four (9.52\%) thyroid hormones, four $(9.52 \%)$ drugs used in benign prostatic hyperplasia, two $(4.76 \%)$ antipsychotics, two (4.76\%) anti-glaucoma drugs and miotics, two $(4.76 \%)$ insulins and analogues, one $(2.38 \%)$ beta-blocking agents, one (2.38\%) digitalis glycosides, one $(2.38 \%)$ organic nitrates and one $(2.38 \%)$ vitamin D and analogues.

Conclusion The RE affected more than half of the patients admitted to the ED. The most prevalent discrepancy was the omission of medication and the drugs most implicated were statins.

\section{REFERENCES AND/OR ACKNOWLEDGEMENTS}

No conflict of interest.

\section{PSQ-164 DRUG-DRUG INTERACTIONS IN PATIENTS WITH CARDIOVASCULAR DISEASES}

${ }^{1} \mathrm{G}$ Lo Surdo, 'E Volpi ${ }^{*},{ }^{1} \mathrm{M}$ Zizevskikh, 'S Tonazzini, 'S Alduini, ${ }^{2} \mathrm{~S}$ Maffei, ${ }^{3} \mathrm{M}$ Baroni, 'S Biagini. 'Fondazione Toscana Gabriele Monasterio, Hospital Pharmacy, Massa, Italy; ${ }^{2}$ Fondazione Toscana Gabriele Monasterio, Division of Clinical And Surgical Heart Diseases, Massa, Italy; ${ }^{3}$ Fondazione Toscana Gabriele Monasterio, Clinical Risk Manager, Massa, Italy

10.1136/ejhpharm-2019-eahpconf.597
Background Medication reconciliation (MedRec) is the process of comparing a patient's medication orders to all of the medications that the patient has been taking. This reconciliation is done to avoid medication errors such as drug interactions. The World Health Organization has recognized MedRec as a recommended standard of quality in health assistance.

Purpose The aim of this analysis was to estimate the prevalence of patients exposed to potentially relevant drug-drug interaction (DDI) at hospital discharge.

Material and methods This was an observational retrospective study involving patients with cardiovascular diseases discharged from our hospital between December 2016 and May 2017.

A total of 1033 patients were included in this study and 8005 drug prescriptions at discharge were analysed $(7.75$ per patient). DDIs were classified as moderate (pharmacological effects must be controlled by individual dose adjustment or on the basis of drug plasma concentration) or severe (drug combination should be avoided in clinical practice).

Results Among 1033 patients included, 271 (26.2\%) were exposed to at least one potential DDI. In particular, 173 patients were discharged with one interaction (16.7\%), 54 patients with two interactions (5.2\%), 23 patients with three interactions $(2.2 \%)$ and 21 were exposed to four or more DDIs (2\%). A total of 445 DDIs were recorded, $75.1 \%$ were classified as moderate and $24.9 \%$ as severe interactions. The median number of DDIs per patients with interactions was 1.6 (range 1-7). The most frequent severe interaction was the combination of some selective serotonin reuptake inhibitors (Paroxetine, Sertraline and Citalopram) and Furosemide $(\mathrm{n}=46 ; 1 \%)$. This combination is known to be associated with an increased risk of cardiotoxicity (QT prolongation and cardiac arrest).

Conclusion From this first analysis, it emerged that one-third of our patients were discharged with at least one potential DDI and a remarkable portion of these combinations was severe. The next step will be to investigate whether adverse clinical events, readmission or death after discharge could be associated with a potentially severe DDI. The final target will be the involvement of a clinical pharmacist within a multidisciplinary team to highlight potential DDIs at discharge and minimise the occurrence of the related risks.

\section{REFERENCES AND/OR ACKNOWLEDGEMENTS}

No conflict of interest.

\section{PSQ-165 QUALITY CONTROL OF INFUSIONS IN PATIENT- SPECIFIC PREPARATIONS FOR ONCOLOGICAL TREATMENT}

${ }^{1} \mathrm{C}$ Vom Eyser*, ${ }^{2} \mathrm{LMH}$ Reinders, ${ }^{2} \mathrm{M}$ Klassen, ${ }^{2} \mathrm{~T}$ Teutenberg, ${ }^{1} \mathrm{~J}$ Tuerk. ${ }^{1}$ Institut Für EnergieUnd Umwelttechnik E.V., Environmental Hygiene and Micropollutants, Duisburg, Germany; ${ }^{2}$ Institut Für Energie- Und Umwelttechnik E.V., Research and Miniaturisation, Duisburg, Germany

\subsection{6/ejhpharm-2019-eahpconf.598}

Background Patient-specific preparations have become a central therapy concept in oncological treatment. The highly potent cytostatic agents are characterised by a narrow therapeutic range. Therefore, exact dosage is important, as lower amounts reduce the effectiveness and higher doses increase the risk of severe side effects. Compound confusion can even result in 
fatal consequences. Missing dependent and independent controls regarding concentration and identity pose a risk for patient safety.

Purpose We developed a concept for a two-stage quality control of the infusion solutions. Drug identity and concentration can be checked onsite after preparation using a combined UVand Raman spectrometer (UV-Raman). This is complemented by an independent method using liquid chromatography coupled to UV detection (HPLC-UV).

Material and methods Methods for the analysis of seven cytostatic drugs and two monoclonal antibodies were developed and validated on an i-QCRx UV-Raman system (B and W Tek Europe GmbH, Lübeck, Germany) and on an Agilent 1200 series HPLC-UV system (Agilent Technologies, Waldbronn, Germany). Sample transport and preparation were evaluated to ensure valid results. In a pilot study we analysed samples from different pharmacies in both systems.

Results Method development and validation were successful for the investigated compounds in both systems. HPLC-UV is more sensitive than UV-Raman. However, due to the content of the preparations, real samples had to be diluted before applying HPLC-UV analysis. Sensitivity of the UV-Raman spectrometer fits to the required concentration range without further dilution. All methods showed reproducible results, UVRaman varied by $0.44 \%$ in a repeated analysis $(n=3)$ of 5 -fluorouracil, while HPLC-UV varied by $0.14 \%$. Results of the investigated samples were also equivalent. In a sample containing paclitaxel with a target concentration of $0.72 \mathrm{mg} / \mathrm{mL}$ we determined $0.73 \mathrm{mg} / \mathrm{mL} \quad(101 \%)$ using UV-Raman and $0.69 \mathrm{mg} / \mathrm{mL}(96 \%)$ using HPLC-UV, for example.

Conclusion UV-Raman and HPLC-UV are suitable for determining the content of patient-individual preparations, both with individual assets and drawbacks. The study showed that the two-stage control concept is appropriate to ensure a highquality level for patient-individual preparations.

\section{REFERENCES AND/OR ACKNOWLEDGEMENTS}

No conflict of interest.

\section{Section 6: Education and Research}

\section{ER-001 HIGH VERSUS LOW DOSE OF URSODEOXYCHOLIC ACID FOR THE MANAGEMENT OF INTRAHEPATIC CHOLESTASIS OF PREGNANCY: A COHORT RETROSPECTIVE STUDY OF MATERNAL AND NEONATAL OUTCOMES}

${ }^{1} \mathrm{R}$ Barazi* ${ }^{*}{ }^{2} \mathrm{O}$ Alkhozondar, ${ }^{1} \mathrm{~A}$ Kilani, ${ }^{3} \mathrm{~S}$ Kassim, ${ }^{4} \mathrm{E}$ Mohamed, ${ }^{5} \mathrm{G}$ La Monica, ${ }^{1}$ E Alhamoud, ${ }^{1} \mathrm{R}$ Elenany, ${ }^{3} \mathrm{M}$ Alloub. ${ }^{1}$ Hamad Medical Corporation, Pharmacy, Al Wakra, Qatar; ${ }^{2}$ Hamad Medical Corporation, Pharmacy, Wakra, Qatar; ${ }^{3}$ Hamad Medical Corporation, Ob/Gyn, Al Wakra, Qatar; ${ }^{4}$ Hamad Medical Corporation, Pharmacy, Dohaq, Qatar; ${ }^{5}$ Sidra Medicine, Ob/Gyn, Doha, Qatar

\subsection{6/ejhpharm-2019-eahpconf.599}

Background Intrahepatic cholestasis of pregnancy (ICP) is a pregnancy-related reversible hepatic disease. The clinical importance of ICP lies in neonatal and maternal ICP-associated complications which include higher rates of perinatal morbidity and mortality, increased rates of caesarean sections, increased risk of meconium staining of amniotic fluid, preterm delivery, fetal bradycardia, fetal distress and fetal demise. The underlying mechanisms associated with poor neonatal outcome have been shown to be associated with elevated maternal total serum bile acids $(40 \mathrm{mmol} / \mathrm{L})$ antenatally.

Ursodeoxycholic acid (UDCA) has shown to result in a significant improvement in symptomatic relief, biochemical markers and gestational age of delivery in patients with ICP. However, a consensus is lacking for the optimal UDCA dosing regimen.

Purpose The study is primarily to compare the effect of a high versus low dose of ursodeoxycholic acid in maternal and neonatal outcomes. This study will also determine the characteristics associated with ICP in a cohort of patients.

Material and methods Design: Retrospective cohort study as ICP is a rarely occurring hepatic disease.

Setting: Most ICP patients get diagnosed or referred to governmental hospitals located in their area of residence for inpatient and outpatient care.

Participants: ICP patients who underwent management of their disease in Ob/Gyn units between July 2016 and July 2017. Patients were identified using institutional medical records.

Main outcome measures: Maternal outcomes: Mode of delivery, gestational age at diagnosis and gestational age at delivery. Neonatal outcomes: APGAR score: $1 \mathrm{~min}, 5 \mathrm{~min}$ and 15 min: birthweight in $\mathrm{g}$ and NICU admission.

Results None of the patients had a history or concurrent diagnosis of other hepatic or biliary disease. A small proportion of both the high-dose and low-dose study population had histories of ICP in previous pregnancies: three in the high-dose group and two in the low-dose group. The mean bile acid level upon diagnosis was $19.7 \mathrm{mmol} / \mathrm{L}$ in the high-dose group paralleled to $17 \mathrm{mmol} / \mathrm{L}$ in the low-dose group. Other neonatal and maternal outcomes will be presented in the poster.

Conclusion This study failed to detect or prove the difference in the maternal and neonatal clinical outcomes between the UDCA high- and low-dose groups.

\section{REFERENCES AND/OR ACKNOWLEDGEMENTS}

https://www.ncbi.nlm.nih.gov/pubmed/24901263https://www. jogc.com/article/S1701-2163(15)30544-2/pdf

No conflict of interest.

\section{ER-002 EFFICACY OF A FIXED-RATIO COMBINATION OF INSULIN DEGLUDEC AND LIRAGLUTIDE IN THE TREATMENT OF TYPE 2 DIABETES MELLITUS}

${ }^{1} \mathrm{~V}$ Ožana*, ${ }^{2} \mathrm{M}$ Frydrych. 'Hospital with Polyclinic Karvina-Raj- Orlova City Branch, Pharmacy, Orlova, Czech Republic; ${ }^{2}$ University of Veterinary and Pharmaceutical Sciences Brno, Department of Human Pharmacology and Toxicology- Faculty of Pharmacy, Brno, Czech Republic

\subsection{6/ejhpharm-2019-eahpconf.600}

Background Based on the current recommendations, a fixedratio combination of insulin degludec and GLP-1 agonist liraglutide (IDegLira) is considered to be an equivalent alternative to an intensified insulin regimen for type 2 diabetes mellitus (T2DM). As a once-daily injection with effects on both fasting and postprandial hyperglycaemia, IDegLira provides, according to several studies, optimal glycemic and metabolic control.

Purpose To determine the effectiveness of IDegLira in the reduction of glycemic parameters, bodyweight and lipid profile parameters in patients with a diagnosis of T2DM. 